Protocols

\title{
Neutral PEGylated liposomal formulation for efficient folate-mediated delivery of MCL1 siRNA to activated macrophages
}

\author{
Eugénia Nogueira $^{\mathrm{a}, \mathrm{b}, 1}$, Jaime Freitas ${ }^{\mathrm{c}, \mathrm{d}, 1}$, Ana Loureiro ${ }^{\mathrm{a}, \mathrm{b}}$, Patrícia Nogueira ${ }^{\mathrm{c}, \mathrm{d}}$, \\ Andreia C. Gomes ${ }^{\mathrm{b}}$, Ana Preto ${ }^{\mathrm{b}}$, Alexandre M. Carmo ${ }^{\mathrm{c}, \mathrm{d}, \mathrm{e}}$, Alexandra Moreira ${ }^{\mathrm{c}, \mathrm{d}}$, \\ Artur Cavaco-Paulo ${ }^{\text {a,* }}$ \\ a CEB - Centre of Biological Engineering, University of Minho, Braga, Portugal \\ b CBMA - Centre of Molecular and Environmental Biology, Department of Biology, University of Minho, Braga, Portugal \\ ${ }^{c}$ I3S - Instituto de Investigação e Inovação em Saúde, Universidade do Porto, Portugal \\ d IBMC - Instituto de Biologia Molecular e Celular, Rua do Campo Alegre 823, 4150-180 Porto, Portugal \\ e ICBAS - Instituto de Ciências Biomédicas Abel Salazar, Universidade do Porto, Rua Jorge Viterbo Ferreira, 228, 4050-313 Porto, Portugal
}

\section{A R T I C L E I N F O}

\section{Article history:}

Received 30 September 2016

Received in revised form 7 April 2017

Accepted 10 April 2017

Available online 13 April 2017

\section{Keywords:}

Activated macrophages

Folate

Liposomes

Neutral

PEGylated

RNA interference

\begin{abstract}
A B S T R A C T
Cationic liposomes are efficient vectors for systemic delivery of therapeutic small interfering RNA (siRNA), taking advantage of RNA interference (RNAi), a naturally occurring gene-silencing mechanism in mammalian cells. However, toxicity at high concentrations, short circulating half-lives and lack of specificity restrict their successful application in a wider scale. The purpose of this study was to evaluate the efficiency of neutral liposomes containing polyethylene glycol (PEG) to encapsulate siRNA in their aqueous core. This formulation will reduce drastically the toxicity associated to cationic liposomes by bringing surface charge to almost zero, increasing stealth degree and therefore circulation time. In this study, we evaluate the efficiency of folate-targeted liposomes for specific delivery of siRNA to activated macrophages, key effector cells in rheumatoid arthritis (RA) pathology which specifically express folate receptor $\beta$ (FR $\beta$ ). Myeloid cell leukaemia-1 (Mcl-1) is a protein essential for synovial macrophage survival, since Mcl-1 suppression results in the induction of apoptosis. The effect of MCL1 siRNA incorporated in liposomal formulation was assessed in primary human macrophages and successful inhibition of Mcl-1 expression was achieved. Here we show that the neutral liposomal derived from DOPE (1,2-dioleoylsn-glycero-3-phosphoethanolamine) formulation developed is efficient to encapsulate MCL1 siRNA and silencing gene expression in activated human macrophages.
\end{abstract}

(c) 2017 Elsevier B.V. All rights reserved.

\section{Introduction}

RNAi is a naturally occurring gene-silencing mechanism that controls endogenous gene expression of in a sequence-specific manner at the messenger RNA (mRNA) level [1]. Double-stranded RNA is processed into small interfering RNA (siRNA), which is a 21 nucleotides long molecule, and then incorporated into a silencing complex which targets and silences mRNA with complementary sequences [2]. However, to achieve an in vivo application for siRNAs important issues still remain to be solved. Highly anionic

\footnotetext{
* Corresponding author at: CEB - Centre of Biological Engineering, University of Minho, Campus of Gualtar, 4710-057 Braga, Portugal.

E-mail addresses: artur@deb.uminho.pt, artur@det.uminho.pt (A. Cavaco-Paulo).

1 These authors contributed equally to this work.
}

oligomeric siRNA molecules cannot cross the barriers of biological membranes and are prone to nuclease mediated degradation in cellular environments [3]. Therefore, there is the need to increase the efficiency of systemic delivery of siRNAs, which has been partially achieved by the development of novel delivery systems. Among these, the cationic liposomal vectors have received significant attention, although some disadvantages, including toxicity at higher concentrations, have restricted their widespread application [4].

For lipid-based non-viral vectors to be useful in nucleic acids delivery, they must fulfill several requirements [5]. Nucleic acid carriers should comprise a nucleic acid-containing core in a lipid envelope layer enabling intracellular delivery and processing. Also, a "stealth layer" should be incorporated to ensure biological stability reflected in adequate circulation times. Finally, the carriers should contain a targeting layer to enhance the particle uptake into specific target cells. It was demonstrated that mixing siRNA with 
preformed highly PEGylated cationic liposomes results in siRNAliposome complexes that instantaneously release their siRNA when in contact with full human serum. Further characterization demonstrated that most of the siRNA was bound to the outer surface of the liposomes [6].

$\mathrm{RA}$ is a common chronic inflammatory rheumatic disease, affecting almost $1 \%$ of the world population [7]. This autoimmune disease is characterized by inflammation of the joints, resulting in synovial hyperplasia by infiltration of activated immune cells further leading to cartilage and bone destruction [8]. Macrophages are critical in the pathogenesis of RA [9] and resistance to apoptosis is an important factor to their persistence in the rheumatoid joint [10]. FR $\beta$, whose expression is particularly elevated in RA synovial macrophages [11], has been used as a target for immunotherapy in a number of clinical conditions. However, due to their intrinsic phagocytic capacity, it is challenging to achieve a macrophage-specific delivery.

In vivo utilization of siRNA as a therapeutic approach in rheumatoid diseases was previously demonstrated by electro transfer of siRNA targeting Tumor Necrosis Factor $\alpha$ (TNF- $\alpha$ ) in arthritic joint tissue of mice and rats [12]. However, this local approach may be difficult to apply at the clinical setting [13]. Mcl-1, a highly regulated member of the anti-apoptotic B-cell lymphoma-2 (Bcl-2) family of proteins, is essential for synovial macrophage survival. It has been reported that Mcl-1 is highly expressed in the rheumatoid joint, particularly in macrophages [14]. Mcl-1 suppression results in the induction of apoptosis [15], suggesting that may be used as a potential therapeutic target in RA.

This work aims to specifically apply PEGylated, DOPE-derived neutral liposomes, as delivery agent for siRNA to activated macrophages. Anchoring folate (or folic acid, FA) at the liposomal surface leads to the liposomes to be targeted to FR $\beta$ expressed at the surface of macrophages. Locked nucleic acids (LNAs), chemically modified siRNAs, were used as an alternative to classic siRNA molecules, as LNAs have been demonstrated to be more stable and more resistant to degradation by RNases compared to other siRNA molecules [16]. MCL1 LNA was used to silence the expression of this key gene in macrophage survival. Finally, the analysis of the effect of MCL1 targeting by the LNA-loaded liposomes was performed by evaluating levels of Mcl-1 protein expression and apoptosis induction.

\section{Materials and methods}

\subsection{Materials}

DOPE and $N$-(carbonyl methoxypolyethylene glycol-2000)1,2-distearoyl-sn-glycero-3-phosphoethanolamine (DSPE-MPEG) were obtained from Lipoid GmbH (Germany). Cholesterol $(\mathrm{CH})$ was obtained from Sigma (USA). LNAs labeled with fluorescein (FAM, $\lambda e x=495 \mathrm{~nm}, \lambda e m=520 \mathrm{~nm}$ ) were purchased from Exiqon (Denmark). The sequences of LNAs were as follows: 5'- TTCCTGATGCCACCTT/36-FAM $-3^{\prime}$, for MCL1 and 5'GTGTAACACGTCTATACGCCCA/36-FAM - 3', for negative control (NC). Throughout all experiments, RNase-free materials and conditions were carefully applied.

\subsection{Preparation of LNA loaded liposomes}

Liposomes composed of DOPE/CH/DSPE-MPEG (54:36:10, molar ratio) were prepared using HYDRA protocol [17]. Briefly, known amounts of DOPE, CH and DSPE-MPEG were dissolved in chloroform in a $50 \mathrm{~mL}$ round-bottom flask. The organic solvent was evaporated using a rotary evaporator. The resultant dried lipid film was dispersed in phosphate-buffered saline (PBS) buffer contain- ing Folate-peptide (0.75 molar ratio) and $10 \mu \mathrm{M}$ LNAs. The lipid film with the LNA was allowed to hydrate overnight at $4{ }^{\circ} \mathrm{C}$, resulting in LNA loaded liposomes. Thereafter, the mixture was extruded (extruder supplied by Lipex Biomembranes Inc.) through $200 \mathrm{~nm}$ and $100 \mathrm{~nm}$ pore size polycarbonate filters (Nucleopore) to form large unilamellar vesicles. After preparation, eventual free LNA was separated using Amicon Ultra Centrifugal Filter Units (100 kDa cut-off), which demonstrated have low binding LNA ability. The efficiency of encapsulation of LNA in the liposomal formulation was measured by fluorescence spectroscopy, through their FAM labeling. The possible interaction of LNA with FA-peptide was discarded by use of untargeted liposomes, with similar encapsulation efficiency.

\subsection{Determination of the zeta potential and size distribution}

The zeta-potential ( $\zeta$-potential) values and size distribution of liposomes were determined at $\mathrm{pH} 7.4$ (PBS buffer) and $25.0^{\circ} \mathrm{C}$ by electrophoretic laser Doppler anemometry and photon correlation spectroscopy (PCS), respectively, using a Malvern Zetasizer NS (Malvern Instruments). The lipid concentration was held constant at $400 \mu \mathrm{M}$. The viscosity and refractive index values were $0.890 \mathrm{cP}$ and 1.330 , respectively.

\subsection{Cell culture conditions}

Human peripheral blood mononuclear cells were isolated from buffy coats of healthy blood donors from the Immunohaemotherapy Department of Hospital de São João(Porto, Portugal) by centrifugation over Lymphoprep (Axis-Shield), and monocytes were further purified by magnetic cell sorting using CD14 microbeads (Miltenyi Biotec). The ethics committee granted approval for the study and all volunteers gave their informed consent. Monocyte-derived macrophages were generated in RPMI 1640 (supplemented with 10\% fetal calf serum (FCS), $2 \mathrm{mM} \mathrm{L-}$ glutamine, $100 \mathrm{U} / \mathrm{mL}$ penicillin and $100 \mu \mathrm{g} / \mathrm{ml}$ streptomycin). M1 and M2 macrophages were obtained by differentiation of monocytes with granulocyte-macrophage colony-stimulating factor (GM-CSF, $20 \mathrm{ng} / \mathrm{ml}$ ) or M-CSF $(50 \mathrm{ng} / \mathrm{ml})$ for 7 days, respectively. M1 macrophages were activated with lipopolysaccharide (LPS, $100 \mathrm{ng} / \mathrm{ml}$ ) and interferon (IFN)- $\gamma(25 \mathrm{ng} / \mathrm{ml}$ ) and M2 macrophages were activated with interleukin (IL)-4 $(20 \mathrm{ng} / \mathrm{ml})$ or IL-10 $(10 \mathrm{ng} / \mathrm{ml})$ for 2 days. Raw 264.7 cells were routinely cultivated in RPMI 1640 supplemented with $10 \%$ FCS, 2 mM L-glutamine, $100 \mathrm{U} / \mathrm{mL}$ penicillin and $100 \mu \mathrm{g} / \mathrm{ml}$ streptomycin.

\subsection{RNA isolation, CDNA synthesis and quantitative RT-PCR}

Total RNA was extracted using the TRIzol Reagent (Invitrogen) in conjunction with the PureLink RNA Mini Kit (Ambion), according to the manufacturer's instructions, and quantified with a NanoDrop ND-1000 spectrophotometer (Nanodrop Technologies). cDNA synthesis was performed with random hexamers and Superscript III following the manufacturer's instructions (Invitrogen). cDNA was then quantified in an iQ5 Real-Time PCR system (Bio-Rad) with iQ SYBR Green Supermix (BioRad). Oligonucleotide primers used for quantitative Real-Time PCR (qPCR): forward, 5'- CAAAACGGGACTGGCTAGTTAAA -3', reverse, 5'- CCTTCTAGGTCCTCTACATGGAAGA -3', for human MCL1, forward, 5'- TGTTCTTGTAAGGACGAAAC -3', reverse, 5' TTAAGAGTCCCCTATTGCAC - 3', for mice Mcl- 1 , and forward, $5^{\prime}$ TGCTATCCAGGCTGTGCTATCC $-3^{\prime}$, and reverse, 5'- CCAGGTCCAGACGCAGGATG $-3^{\prime}$, for $\beta$-actin. $\beta$-actin gene was used as the internal reference. Data were analyzed using iQ5 Optical System software (Bio-Rad). 


\section{6. siRNA delivery}

LNA transfection was performed using GenMute siRNA Transfection Reagent for Primary Macrophages (SignaGen) following the manufacturer's recommendations. Macrophage exposure to FRtargeting liposomes was performed as previously reported [18]. Briefly, cells were incubated with $100 \mathrm{nM}$ of LNA-loaded liposomes in complete medium at $37^{\circ} \mathrm{C}$. After incubation, cells were washed twice with PBS ( $\mathrm{pH} 7.4$ ) and then resuspended in PBS for fluorescence-activated cell sorting (FACS) analysis or TRIzol Reagent (Invitrogen) for qPCR.

\subsection{Western blot}

Cells were collected, washed with PBS and resuspended in lysis buffer (10 mM Tris- $\mathrm{HCl} \mathrm{pH}$ 7.4, $150 \mathrm{mM} \mathrm{NaCl}, 1 \mathrm{mM}$ EDTA and 1\% $\mathrm{v} / \mathrm{v}$ : NP-40) containing $1 \mathrm{mM}$ phenylmethylsulfonyl fluoride and complete protease inhibitor cocktail (Roche Diagnostics $\mathrm{GmbH}$ ). After $30 \mathrm{~min}$ of incubation on ice, the suspensions were centrifuged and the supernatants were collected. Protein concentrations were measured by Bradford reagent (Sigma-Aldrich). Equal amounts of each protein sample were separated by $10 \%$ SDS-polyacrylamide gel electrophoresis (SDS-PAGE) and transferred to a nitrocellulose membrane using iBlot Dry Blotting System (Invitrogen). The membranes were incubated at room temperature for $60 \mathrm{~min}$ in blocking buffer containing $5 \%$ fat-free milk and TBS-T (20 mM Tris-HCL pH $7.6,137 \mathrm{mM} \mathrm{NaCl}, 0.1 \%$ Tween 20 ) and then probed overnight at $4{ }^{\circ} \mathrm{C}$ with primary mouse monoclonal antibodies specific for Mcl-1 (1:5000, Ab22, eBioscience) diluted in 3\% fat-free milk in TBS-T. Following washes with TBS-T, membranes were incubated with goat anti-mouse IgG-HRP secondary antibody (1:25000, Santa Cruz Biotechnology, Inc.) diluted in TBS-T for $1 \mathrm{~h}$ at room temperature. Next, the membranes were washed again and submitted to WesternBright ${ }^{\mathrm{TM}}$ Quantum (Advansta) detection system for 5 min before being developed by chemiluminiscence. In order to re-probe the membrane to analyze the expression of a constitutive protein (as loading control), membranes was submitted to a stripping protocol $\left(\right.$ Abcam $\left.^{\circledR}\right)$ with a mild stripping buffer, prepared according to manufacturer's instructions. After stripping, membranes were blocked again, incubated with mouse anti-human $\alpha$-Tubulin antibody (1:30000, Sigma-Aldrich, clone B-5-1-2) or $\beta$-actin (1:1000, Abcam $^{\circledR}$ ), and then incubated with the secondary antibody and developed, as described before.

\subsection{Apoptosis assay}

The percentage of apoptotic cells was determined by Annexin V-FITC/Propidium Iodide staining according to the manufacturer's instruction (ExBio). Briefly, cells from each sample were washed in PBS and resuspended in Annexin V binding buffer $(100 \mu \mathrm{L})$. Annexin V-FITC $(5 \mu \mathrm{L})$ and Propidium Iodide $(5 \mu \mathrm{L})$ was added to each cell suspension, gently mixed and incubated for $10 \mathrm{~min}$ at room temperature. Finally, apoptotic (Annexin Vpos $/ \mathrm{PI}^{\text {neg }}$ ) and dead cells (double positives) were assessed by flow cytometry (BD FACS CANTO II).

\section{Results and discussion}

Although cationic liposomes are among the most popular nucleic acid delivery agents, some concerns remain regarding their safety for therapeutic use. In particular, toxicity of certain cationic lipid particles has been reported both in vitro and in vivo.

Additionally, it was demonstrated that simply mixing siRNA with preformed highly PEGylated liposomes does not avoid siRNA release into the serum and subsequent degradation by nucleases
Table 1

Physicochemical characterization of FR-targeting liposomal formulations based on DOPE/CH/DSPE-MPEG. Values represent the mean \pm SD of 3 independent experiments.

\begin{tabular}{lccc}
\hline LNA & Z-average $(\mathrm{nm})$ & Polydispersity index $(\mathrm{PdI})$ & $\zeta$-Potential $(\mathrm{mV})$ \\
\hline- & $122.1 \pm 2.44$ & $0.048 \pm 0.019$ & $-0.945 \pm 1.18$ \\
MCL1 & $120.9 \pm 2.53$ & $0.050 \pm 0.017$ & $-0.993 \pm 1.24$ \\
\hline
\end{tabular}

[17]. To be optimally protected siRNA molecules need to be encapsulated inside the core of highly PEGylated liposomes, as siRNAs that are bound to the outer surface are almost instantly released in serum through competition with ubiquitous negatively charged serum components. Efficient gene silencing has been shown by liposomes made of the neutral DOPC (1,2-dioleoyl-sn-glycero-3phosphocholine) without surface modification to improve stealth or targeting, being the siRNA incorporated into DOPC $[19,20]$.

We have previously described an innovative strategy for targeted liposomal delivery that uses a hydrophobic fragment of a surfactant protein conjugated to a folate moiety and a linker, the SP-DS3 peptide [21]. We have shown that this is more efficient than other classical systems using the PEG linker [21]. The novel liposomal constructs present high stealth degree, achieved with 10\% DSPE-MPEG. Moreover, they present low cytotoxicity (at least until $750 \mu \mathrm{g} / \mathrm{ml}$ and $72 \mathrm{~h}$, Fig. S1 ) [21] and are able to deliver drugs to activated macrophages while not affecting normal cells, both in vitro and in vivo [18]. We measured the uptake of these FRtargeting liposomes in the monocytic cell line THP- 1 with and without overexpression of human FR $\beta$. Liposomes with FA were highly internalized by THP-1 cells retrovirally transformed with FR $\beta$ in comparison with the wild-type THP-1 cells, which weakly express FR $\beta$, that showed minimal uptake [18]. Furthermore, in comparison to liposomes without FA, targeted liposomes strongly accumulated in the joints of arthritic mice [18].

We propose to use DOPE-derived neutral PEGylated liposomes containing FA ligand at their surface, to promote specific MCL1 LNA delivery in activated macrophages, as a potential tool for treating RA.

\subsection{Characterization of liposome encapsulating LNA}

Size and surface charge are important characteristics in the successful development of vectors for systemic delivery. The size distribution of LNA-loaded liposomes was determined by dynamic light scattering (DSL) measurements. The results indicated that the size distribution pattern of liposomes was not influenced by encapsulation of MCL1 LNA (similar results obtained to NC LNA, data not shown), as in both cases a size of approximately $120 \mathrm{~nm}$ was observed (Table 1). Furthermore, both liposomal formulations exhibited a narrowed size distribution (polydispersity index $<0.1$ ), which is very favourable for the purpose of our work (Table 1 ). The $\zeta$-potential (electrical potential at the surface of the hydrodynamic shear around the colloidal particle) was also evaluated. The surface potential of the particles cannot be measured directly, thus the $\zeta$ potential is usually determined as a characteristic parameter for the particle charge. Similar to empty liposomes, the formulation prepared with LNA presented a $\zeta$-potential close to zero (Table 1 ), suggesting that LNAs are encapsulated in the aqueous interior of liposomes, not altering the formation of neutral liposomes. These physicochemical characteristics of liposomal formulations remain unaltered at least until 16 weeks, suggesting a high stability of these liposomes in terms of size distribution.

Surprisingly, the encapsulation efficiency of $\approx 100 \%$ for MCL1 LNA in neutral liposomes indicates that, by hydrating the lipid film with a concentrated solution containing the LNA (HYDRA protocol), the negative charges on the LNA come into contact with the 


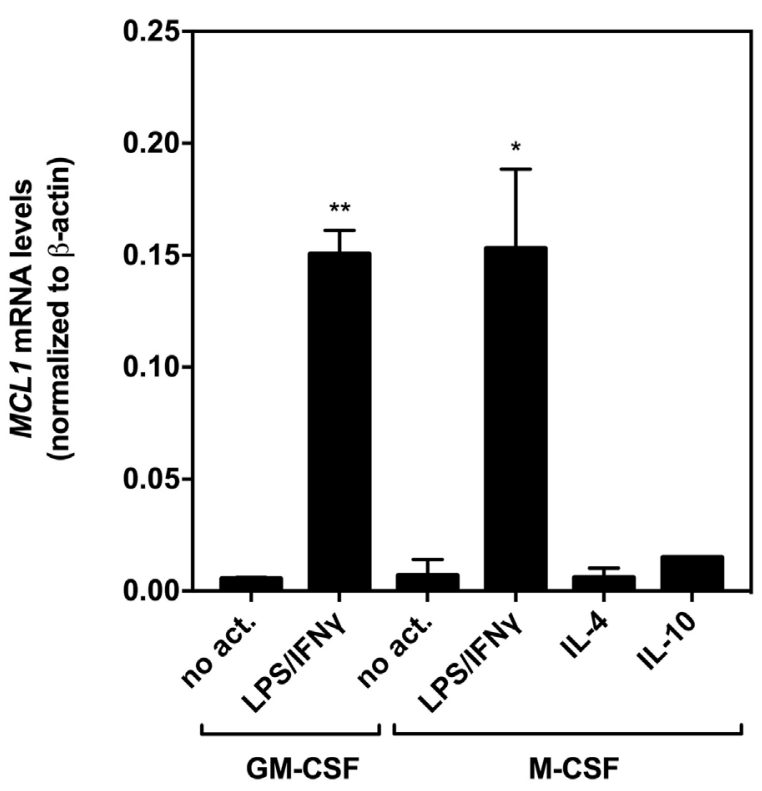

Fig. 1. MCL1 expression levels in macrophages. Primary human macrophages were obtained by differentiation of monocytes with GM-CSF or M-CSF for 7 days followed by $48 \mathrm{~h}$ activation with different stimuli. Relative mRNA expression levels were quantified by RT-qPCR and normalized for $\beta$-actin. Data show the mean + standard deviation for at least three independent experiments. Comparisons were performed using an unpaired two-tailed $t$-test $\left({ }^{*} p<0.05\right.$ and $\left.{ }^{*} p<0.005\right)$.

neutral charge of all liposomal formulation, prior to liposome formation. Indeed, as soon as the liposomes form closed vesicles, the LNA will not be able to penetrate the lipid bilayers anymore. This alternative to the classic protocol of mixing siRNA with preformed liposomes allows a good distribution of the negatively charged LNA over all liposomal formulation, because the contact between them occurs before and during the formation, and the final closing of the liposomal membranes. The HYDRA protocol allows a distribution of the LNA molecules through lipid film in the round-bottom flasks, enabling a much larger contact area. The total encapsulation of siRNA molecules inside the core of the highly PEGylated liposomes

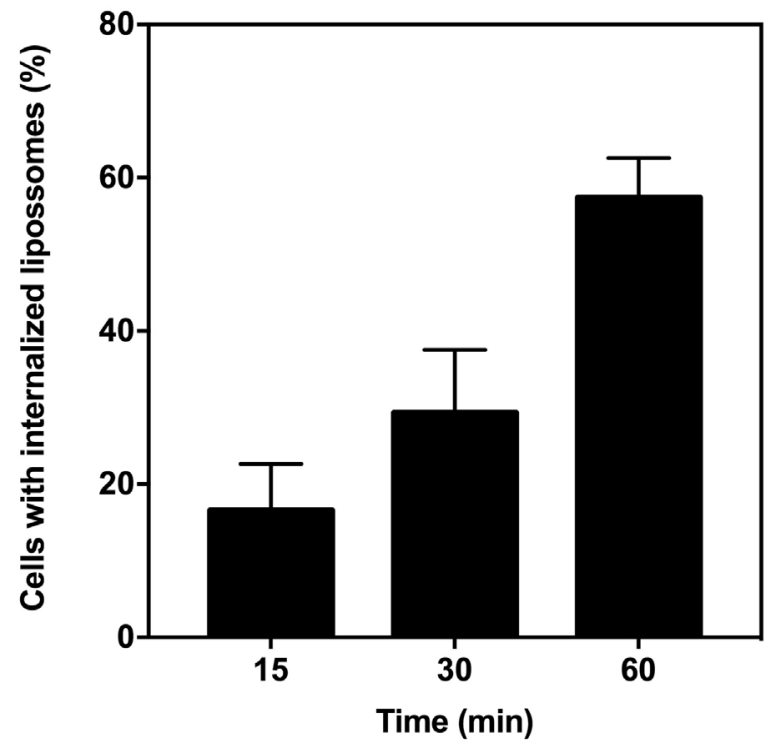

Fig. 2. Uptake of FR-targeting liposomes loaded with MCL1 LNAs by FRß-expressing macrophages. Macrophages were obtained by incubation with $50 \mathrm{ng} / \mathrm{ml} \mathrm{M-CSF}$ for 7 days followed by 1 day of activation with $20 \mathrm{ng} / \mathrm{ml}$ IL-4. These FR $\beta$-expressing macrophages were incubated with $100 \mu \mathrm{g} / \mathrm{ml}$ of liposomes (FAM-conjugated) for 15,30 and $60 \mathrm{~min}$ at $37^{\circ} \mathrm{C}$. Internalization of particles was analyzed by ImageStream multispectral imaging flow cytometry as previously described [18] (Fig. S2). Data show the mean + standard deviation of two independent experiments.

avoid their released into the serum and subsequent degradation by nucleases.

\subsection{MCL1 expression in macrophages}

MCL1 is expressed in macrophages and is essential for their survival [15]. Macrophages M1 (classical, inflammatory) and M2 (alternative, anti-inflammatory) are referred as the two extremes of a spectrum of possible macrophage activation status [22]. We evaluated the effect of macrophage differentiation on MCL1 expression by qPCR quantification in M1 and M2 macrophages developed
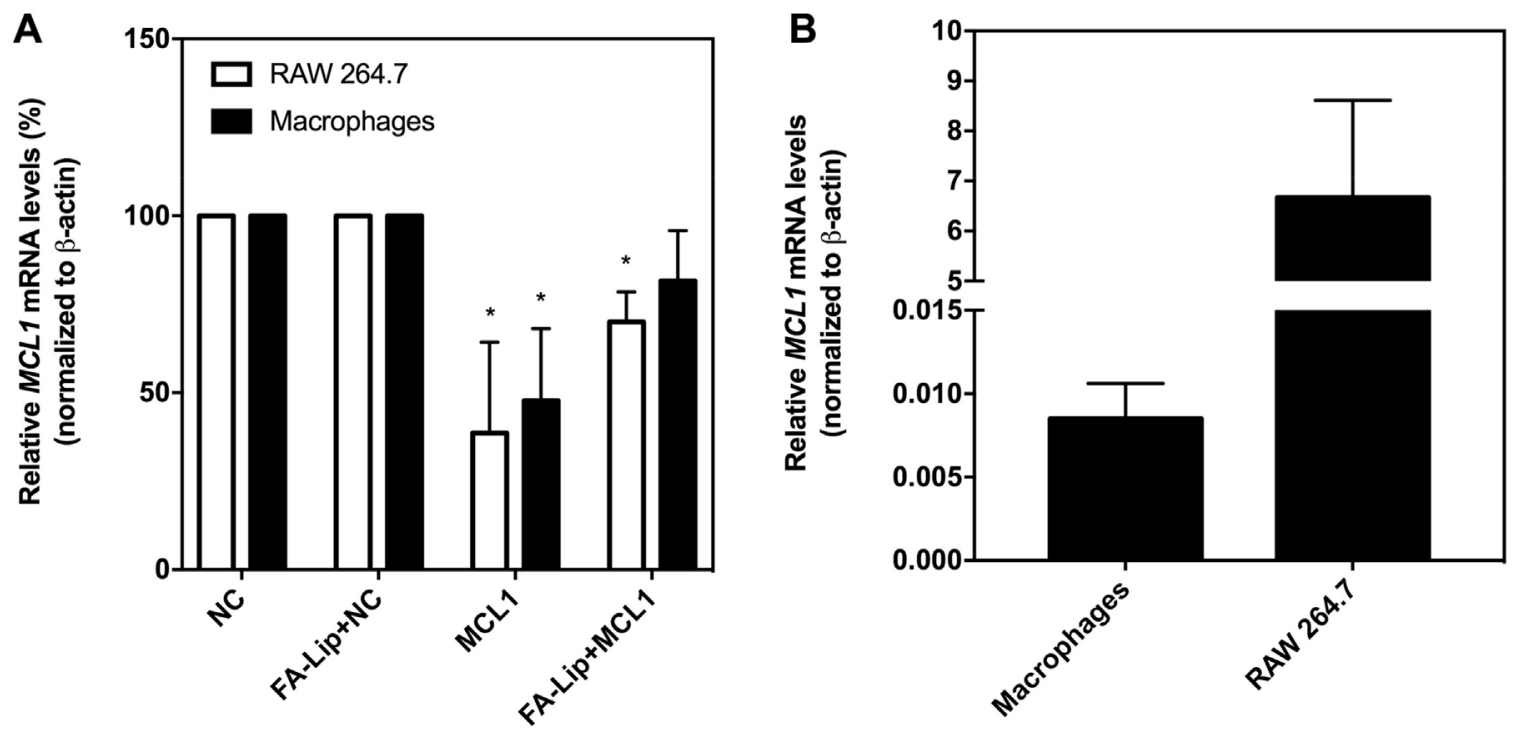

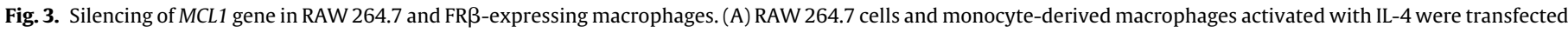

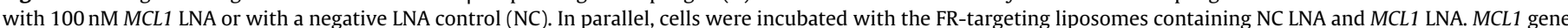

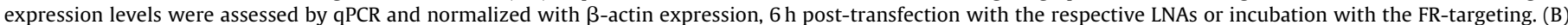

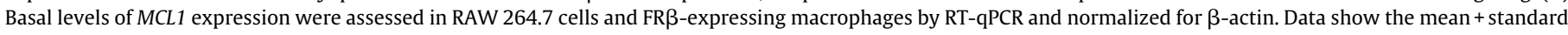
deviation of two independent experiments. Comparisons were performed using an unpaired two-tailed $t$-test $\left({ }^{*} p<0.05\right)$. 
A

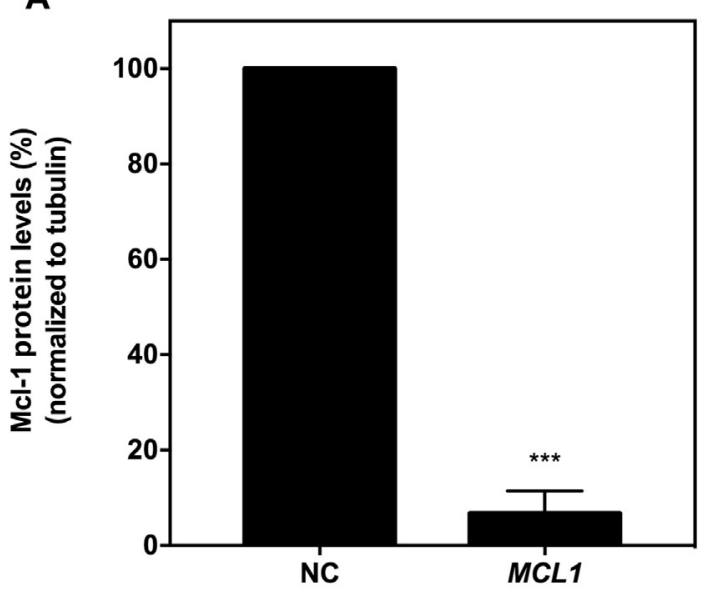

B

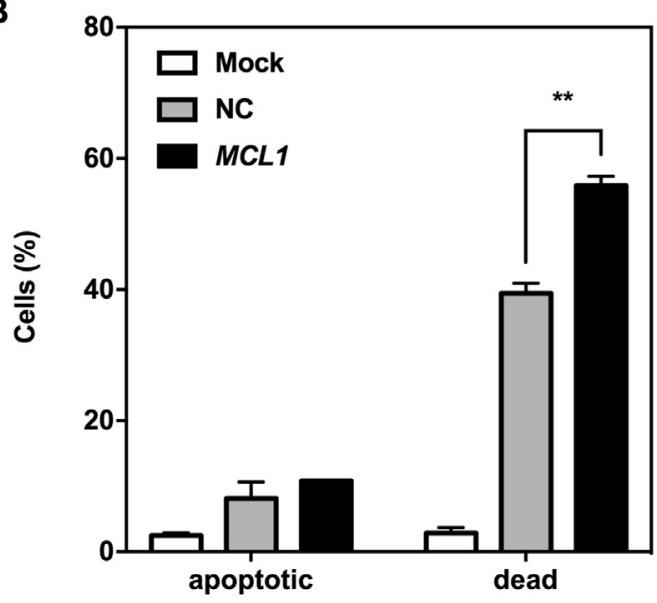

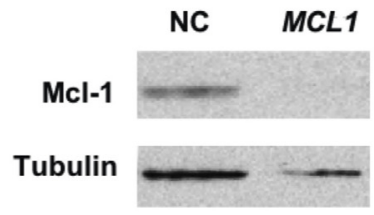

C

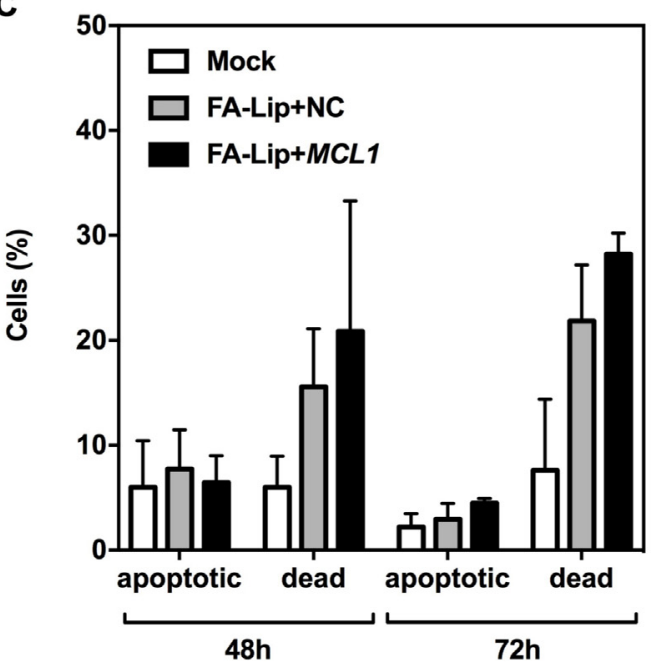

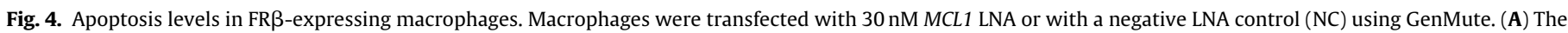

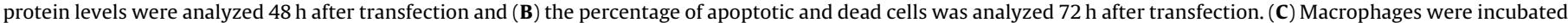

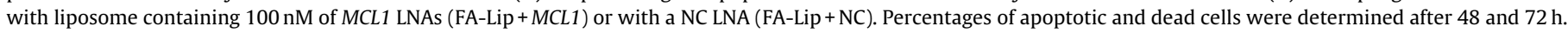

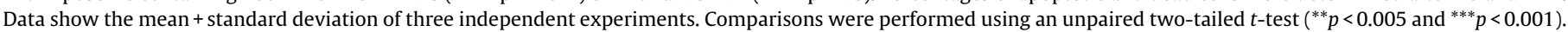

in culture. These were obtained by differentiating primary human monocytes into macrophages using GM-CSF or M-CSF, respectively.

After 7 days, GM-CSF-differentiated macrophages were activated with LPS and IFN- $\gamma$ (simultaneously), while M-CSFdifferentiated macrophages were also activated with IL-4 and IL-10 for $48 \mathrm{~h}$ MCL1 mRNA is shown to be highly expressed after activation with LPS and IFN- $\gamma$, while in IL- 4 or IL-10 activated macrophages the expression is low (Fig. 1). As the acquisition of folate uptake ability is promoted by M-CSF and maintained by IL- 4 [23] and only M-CSF differentiated macrophages express high levels of FR $\beta$, IL-4 stimulated macrophages were chosen and used in subsequent studies.

\section{3. $\mathrm{MCL} 1$ silencing in macrophages}

Previous results shown that liposomes with FA were highly internalized by THP-1 cells (human monocytic cell line) that specifically express FR $\beta$ [18]. To evaluate the efficiency of therapeutically relevant FR-targeting LNA-loaded liposomes, we targeted FR $\beta$ expressing macrophages (M-CSF+IL4) with LNAs against MCL1. These LNA containing FR-targeting liposomes are efficiently internalized by FR $\beta$-expressing macrophages as $\approx 60 \%$ of macrophages internalize liposomes after $1 \mathrm{~h}$ of incubation (Fig. 2).
The release and effect of the LNA from these new liposomes were then tested in primary human macrophages expressing FR $\beta$ and the murine macrophage-like RAW 264.7 cells that express FR $\alpha$ [24]. This cell line was shown to functionally bind and internalize a folate-based nanoparticle evaluated as a therapeutic device for rheumatoid arthritis [25]. Expression of MCL1 and the silencing efficiency of the newly developed liposomal formulation was analyzed by qPCR (Fig. 3A). To assess the effect of the LNA on MCL1 expression in macrophages, LNAs were transfected using the GenMute reagent (SignaGen) and the silencing efficiency quantified by qPCR, in comparison to a negative LNA control (no homology to any known microRNA or mRNA sequences in mouse, rat or human). As expected, transfection of the LNA against the anti-apoptotic $M C L 1$ gene significantly reduced $M C L$ mRNA levels in both cell types ( $\approx 60 \%$ ). Similarly, although to a lower degree, MCL1 mRNA levels were decreased approximately 40\% and 30\%, in RAW 264.7 and macrophages treated with FR-targeting liposomes, respectively. These differences in MCL1 silencing may be due to the high stability of liposomes and the controlled release of the LNA in ex vivo systems. We also observed that although MCL1 mRNA levels are increased in murine RAW 264.7 cells in comparison to human primary macrophages (Fig. 3 B), MCL1 silencing is achieved in both cell types. 


\subsection{In vitro apoptosis levels in macrophages}

Mcl-1 protein expression in macrophages was examined after treatment with MCL1 LNA. The results show a strong decrease in Mcl-1 protein expression (90\%; Fig. 4A) indicating that the MCL1 silencing effect is more clearly observed at the protein levels than at the mRNA level ( $\approx 60 \%$; Fig. 3A). More importantly, the decrease in Mcl-1 expression led to $\approx 70 \%$ of dead cells after $72 \mathrm{~h}$ of transfection (Fig. 4B). As Mcl-1 is an anti-apoptotic member of the B-cell lymphoma 2 (Bcl-2) family of apoptosis-regulating proteins, these results clearly show that MCL1 silencing is functionally relevant.

Levels of apoptosis were then analyzed in FR $\beta$-expressing macrophages incubated with LNA-loaded liposomes (Fig. 4C). These macrophages did not show significant higher levels of apoptosis when incubated for 48 and $72 \mathrm{~h}$ with $100 \mathrm{nM}$ of Mcl-1 LNA-loaded liposomes (corresponding at $\sim 264 \mu \mathrm{g} / \mathrm{ml}$ liposomes). Nonetheless, an increase in the number of dead cells was observed for MCL1 LNA-loaded liposomes in comparison to the control, in particular at $72 \mathrm{~h}$. The lower levels of apoptosis remain even after incubation with $200 \mathrm{nM}$ of MCL1 LNA-loaded liposomes (data not shown). Due to lower levels of apoptosis, Mcl-1 protein expression was not analyzed in macrophages after incubation with MCL1 LNA-loaded liposomes.

The differences observed between free MCL1 LNA and loaded liposomes are most probably due to the slow release of the LNA into the cells, from the very stable liposomes. The lower degree of MCL1 silencing induced by LNA-loaded liposomes may not sufficiently reduce the $\mathrm{Mcl}-1$ protein levels to trigger apoptosis. As previously reported, $M C L 1$ downregulation may not sufficient per se cause apoptosis, but instead, it sensitizes cells to apoptosis [26]. Of note, MCL1 LNA and LNA-loaded liposomes are uptaken by the FR $\beta$ expressing macrophages via different pathways, possibly resulting in different levels of silencing and apoptosis. While MCL1 specific inhibition should induce apoptosis in a Mcl-1-dependent pathway, the cells may also be protected by other anti-apoptotic BCL-2 family members [27]. Furthermore, Mcl-1 is a short-lived protein that undergoes a complex regulation, including transcriptional and translational control, as well as the involvement of different E3 ligases and deubiquitinating enzymes [28].

\section{Conclusion}

In conclusion, here we show that the HYDRA protocol enables encapsulation of MCL1 LNA into FR-targeted, highly PEGylated and neutral liposomal formulation to an extent that attains a maximal encapsulation efficiency of nearly $100 \%$. This protocol is a laboratory-scale method that is a simple and inexpensive alternative to the classic protocol of mixing LNA with preformed liposomes, but with much higher LNA encapsulation efficiency. We show that it was possible to use a DOPE-derived neutral liposomal formulation and thereby reduce drastically the toxicity associated to cationic liposomes (Fig. S1). This neutral liposomal formulation ensures that siRNA molecules are encapsulated inside the core of the highly PEGylated liposomes. In this way, there is no siRNAs bound to the outer surface of liposomes, which would be instantly released in serum through competition with ubiquitous negatively charged serum components and subsequent degraded by nucleases. Furthermore, we also demonstrate that these MCL1 LNA liposomes are efficiently uptaken by FR $\beta$-expressing primary macrophages.

\section{Acknowledgements}

Eugénia Nogueira (SFRH/BD/81269/2011) and Ana Loureiro (SFRH/BD/81479/2011) hold scholarships from Fundação para a
Ciência e a Tecnologia (FCT). This study was funded by the European Union Seventh Framework Programme (FP7/2007-2013) under grant agreement NMP4-LA-2009-228827 NANOFOL. The authors thank the FCT Strategic Project of UID/BIO/04469/2013 unit, the project RECI/BBB-EBI/0179/2012 (FCOMP-01-0124FEDER-027462) and the Project "BioHealth - Biotechnology and Bioengineering approaches to improve health quality”, Ref. NORTE07-0124-FEDER-000027, co-funded by the Programa Operacional Regional do Norte (ON.2-O Novo Norte), QREN, FEDER. This work was also supported by FCT I.P. through the strategic funding UID/BIA/04050/2013. We thank the Immuno-haemotherapy Department of Hospital de São João (Porto, Portugal) for providing buffy coats from healthy volunteers.

\section{Appendix A. Supplementary data}

Supplementary data associated with this article can be found, in the online version, at http://dx.doi.org/10.1016/j.colsurfb.2017.04. 023.

\section{References}

[1] C.C. Mello, D. Conte Jr., Revealing the world of RNA interference, Nature 431 (2004) 338-342

[2] K.A. Whitehead, R. Langer, D.G. Anderson, Knocking down barriers: advances in siRNA delivery, Nat. Rev. Drug Discov. 8 (2009) 129-138.

[3] K. Kumar, B. Maiti, P. Kondaiah, S. Bhattacharya, Efficacious gene silencing in serum and significant apoptotic activity induction by survivin downregulation mediated by new cationic gemini tocopheryl lipids, Mol. Pharm. 1 (2014) 1.

[4] S. Simões, A. Filipe, H. Faneca, et al., Cationic liposomes for gene delivery, Expert Opin. Drug Deliv. 2 (2005) 237-254.

[5] K. Kostarelos, A.D. Miller, Synthetic, self-assembly ABCD nanoparticles; a structural paradigm for viable synthetic non-viral vectors, Chem. Soc. Rev. 34 (2005) 970-994

[6] K. Buyens, B. Lucas, K. Raemdonck, et al., A fast and sensitive method for measuring the integrity of siRNA-carrier complexes in full human serum, J. Controll. Release 126 (2008) 67-76.

[7] R. Sanmarti, V. Ruiz-Esquide, M.V. Hernandez, Rheumatoid arthritis: a clinical overview of new diagnostic and treatment approaches, Curr. Top. Med. Chem. 13 (2013) 698-704.

[8] A.K. Shrivastava, A. Pandey, Inflammation and rheumatoid arthritis, J. Physiol. Biochem. 69 (2013) 335-347.

[9] N. Maruotti, F.P. Cantatore, E. Crivellato, A. Vacca, D. Ribatti, Macrophages in rheumatoid arthritis, Histol. Histopathol. 22 (2007) 581-586.

[10] R.M. Pope, Apoptosis as a therapeutic tool in rheumatoid arthritis, Nat. Rev. Immunol. 2 (2002) 527-535.

[11] N. Nakashima-Matsushita, T. Homma, S. Yu, et al., Selective expression of folate receptor $\beta$ and its possible role in methotrexate transport in synovial macrophages from patients with rheumatoid arthritis, Arthritis Rheum. 42 (1999) 1609-1616.

[12] R.M. Schiffelers, J. Xu, G. Storm, M.C. Woodle, P.V. Scaria, Effects of treatment with small interfering RNA on joint inflammation in mice with collagen-induced arthritis, Arthritis Rheum. 52 (2005) 1314-1318.

[13] M. Khoury, V. Escriou, G. Courties, et al., Efficient suppression of murine arthritis by combined anticytokine small interfering RNA lipoplexes, Arthritis Rheum. 58 (2008) 2356-2367.

[14] H. Liu, P. Eksarko, V. Temkin, et al., Mcl-1 is essential for the survival of synovial fibroblasts in rheumatoid arthritis, J. Immunol. 175 (2005) 8337-8345.

[15] H. Liu, Q. Huang, B. Shi, et al., Regulation of Mcl-1 expression in rheumatoid arthritis synovial macrophages, Arthritis Rheum. 54 (2006) 3174-3181.

[16] O. Mook, J. Vreijling, S.L. Wengel, et al., In vivo efficacy and off-target effects of locked nucleic acid (LNA) and unlocked nucleic acid (UNA) modified siRNA and small internally segmented interfering RNA (sisiRNA) in mice bearing human tumor xenografts, Artif. DNA PNA XNA 1 (2010) 36-44.

[17] K. Buyens, J. Demeester, S.S. De Smedt, N.N. Sanders, Elucidating the encapsulation of short interfering RNA in PEGylated cationic liposomes, Langmuir 25 (2009) 4886-4891.

[18] E. Nogueira, F. Lager, D. Le Roux, et al., Enhancing methotrexate tolerance with folate tagged liposomes in arthritic mice, J. Biomed. Nanotechnol. 11 (2015) 2243-2252

[19] C.N. Landen Jr., A. Chavez-Reyes, C. Bucana, et al., Therapeutic EphA2 gene targeting in vivo using neutral liposomal small interfering RNA delivery, Cancer Res. 65 (2005) 6910-6918.

[20] J. Halder, A.A. Kamat, C.N. Landen Jr., et al., Focal adhesion kinase targeting using in vivo short interfering RNA delivery in neutral liposomes for ovarian carcinoma therapy, Clin. Cancer Res. 12 (2006) 4916-4924.

[21] E. Nogueira, I.C. Mangialavori, A. Loureiro, et al., Peptide anchor for folate-targeted liposomal delivery, Biomacromolecules 16 (2015) 2904-2910. 
[22] J. Li, H.C. Hsu, J.D. Mountz, Managing macrophages in rheumatoid arthritis by reform or removal, Curr. Rheumatol. Rep. 14 (2012) 445-454.

[23] A. Puig-Kroger, E. Sierra-Filardi, A. Dominguez-Soto, et al., Folate receptor beta is expressed by tumor-associated macrophages and constitutes a marker for M2 anti-inflammatory/regulatory macrophages, Cancer Res. 69 (2009) 9395-9403.

[24] Y. Hattori, M. Sakaguchi, Y. Maitani, Folate-linked lipid-based nanoparticles deliver a NFkappaB decoy into activated murine macrophage-like RAW264.7 cells, Biol. Pharm. Bull. 29 (2006) 1516-1520.

[25] T.P. Thomas, S.N. Goonewardena, I.J. Majoros, et al., Folate-targeted nanoparticles show efficacy in the treatment of inflammatory arthritis, Arthritis Rheum. 63 (2011) 2671-2680.
[26] U. Maurer, C. Charvet, A.S. Wagman, E. Dejardin, D.R. Green, Glycogen synthase kinase-3 regulates mitochondrial outer membrane permeabilization and apoptosis by destabilization of MCL-1, Mol. Cell 21 (2006) 749-760.

[27] G. Brumatti, P.G. Ekert, Seeking a MCL-1 inhibitor, Cell Death Differ. 20 (2013) 1440-1441.

[28] S. Varadarajan, M. Vogler, M. Butterworth, et al., Evaluation and critical assessment of putative MCL-1 inhibitors, Cell Death Differ. 20 (2013) 1475-1484. 